\title{
Creation of lateral structures in diblock copolymer thin films during vapor uptake and subsequent drying - Effect of film thickness
}

\author{
A. Sepe ${ }^{\text {a,1 }}$, P. Černoch ${ }^{\text {b }}$, P. Štěpánek ${ }^{\text {b }}$, E.T. Hoppe ${ }^{\text {a }}$, C.M. Papadakis ${ }^{\text {a,* }}$ \\ a Technische Universität München, Physik-Department, Physik weicher Materie, James-Franck-Str. 1, 85748 Garching, Germany \\ ${ }^{\mathrm{b}}$ Institute of Macromolecular Chemistry, Academy of Sciences of the Czech Republic, Heyrovsky Sq. 2, 16206 Prague, Czech Republic
}

\section{A R T I C L E I N F O}

\section{Article history:}

Received 18 July 2013

Received in revised form 30 October 2013

Accepted 5 November 2013

Available online 13 November 2013

\section{Keywords:}

Polymer physics

Grazing-incidence small-angle X-ray

scattering

In situ measurements

Atomic force microscopy

\begin{abstract}
A B S T R A C T
We have studied the structure formation in compositionally nearly symmetric poly(4octylstyrene-b-butylmethacrylate) diblock copolymer films during exposure to saturated vapor of $n$-hexane (a poor solvent for both blocks) and subsequent drying using real-time, in situ grazing-incidence small-angle X-ray scattering (GISAXS). Previous bulk studies revealed a lamellar structure after thermo-annealing; and surface studies on hexane treated samples revealed a lateral surface structure (Černoch et al., 2007) [14]. We focus here on the effect of film thickness which is varied between 1.3 and 2.0 times the bulk lamellar thickness. We report on the lateral repeat distance as well as on the correlation length in the film plane. Complex, non-monotonous behavior is observed for both parameters. Upon drying, the lateral structure created during vapor treatment is stable only for the thick film, not for the intermediate and the thin film. The kinetics depends strongly on the film thickness; especially for the thinnest film, it is very slow. For all film thicknesses, we attempt to identify the most stable morphology, occurring when the inner film structure is close to the one in the bulk.
\end{abstract}

(c) 2013 Elsevier Ltd. All rights reserved.

\section{Introduction}

Thin block copolymer films are promising candidates for a variety of applications, such as the preparation of nanoporous films, nanostructured templates, photonic crystals and biosensors [1,2]. Lateral structures are of special interest, e.g. for their use in organized inorganic substrates $[3,4]$ and for the preparation of nanoporous membranes [5,6]. Solvent vapor induced annealing offers a simple and flexible method to create ordered structures with a potentially high degree of controllability through careful choice of solvent [7-16].

The thin film geometry allows detailed studies of the swelling and rearrangement during vapor treatment,

\footnotetext{
* Corresponding author. Tel.: +49 8928912447.

E-mail address: papadakis@tum.de (C.M. Papadakis).

${ }^{1}$ Present address: University of Cambridge, Department of Physics, Cavendish Laboratory, Madingley Road, Cambridge CB3 OHE, UK.
}

because the structures may be oriented by the film surface and the film/substrate interface. Our previous investigations on lamellar poly(styrene- $b$-butadiene) thin films after spin-coating [17] and during vapor treatment and subsequent drying [15-19] or heat treatment [20] have shown that, using in situ, grazing-incidence small-angle X-ray scattering (GISAXS) with 2D detection, a wealth of information is accessible, such as the lamellar orientation and its distribution as well as the lamellar thickness, i.e. the repeat distance and the number of correlated lamellae. Moreover, information about the lamellar roughness and undulations of the lamellar interfaces can be gained. Combining in situ, real-time GISAXS with atomic force microscopy (AFM), which details the surface morphology, and X-ray reflectometry (XR), which reveals the film thickness as well as possible layered structures inside the film, detailed information on the processes during vapor treatment and drying can be gained giving information 
on their molecular origin. Knowledge of the mechanisms and the kinetics of the processes during solvent vapor annealing plays an important role in the preparation of long-range ordered and regularly oriented structures thus enabling their application.

In the present study, we have examined a poly(4-octylstyrene- $b$-butylmethacrylate) (P(OS- $b$-BMA)) diblock copolymer. It was investigated previously by two of us [14] and it was found that, in the bulk, it forms the lamellar structure after thermo-annealing. $n$-Hexane (HX) - the solvent used for vapor treatment in the present study - dissolves lower and swells higher molar mass fractions of both POS and PBMA homopolymers [14]. It is thus close to non-selective for both blocks, but not a good solvent. Moreover, it was found using AFM that a thin film which has a featureless surface after the preparation forms perpendicular (or standing) lamellae after swelling in HX vapor for a few minutes and subsequent drying [14]. In contrast, acetone vapor treatment resulted in a parallel lamellar orientation. Acetone is a non-solvent for POS and a good solvent for PBMA, thus very selective for PBMA. The quality and selectivity of the solvent for the two blocks thus seem to be key parameters for the final lamellar orientation.

In the present work, the structural changes during vapor treatment of spin-coated films having different film thicknesses were followed using in situ, real-time GISAXS. It was found that treatment with $\mathrm{HX}$ vapor results in the formation of lateral structures, as expected. The processes are complex, and the kinetics depends strongly on the film thickness.

\section{Materials and methods}

Materials: The synthesis and characterization of the poly(4-octylstyrene- $b$-butylmethacrylate) (P(OS-b-BMA)) diblock copolymer are described in Ref. [14]. The overall molar mass is $35600 \mathrm{~g} / \mathrm{mol}$ and the styrene content 62 wt.\%. In bulk, it forms the lamellar morphology with a lamellar thickness $D_{l}^{\text {bulk }}=260 \AA$, as determined using SAXS on a thermo-annealed sample. The glass transition temperatures of the homopolymers are $-40^{\circ} \mathrm{C}$ for POS and $30^{\circ} \mathrm{C}$ for PBMA [14].

Films of three different thicknesses were prepared by spin-coating a $1 \mathrm{wt}$.\% toluene solution onto Si wafers at frequencies of 1000, 2000 and $3000 \mathrm{rpm}$. The wafers had previously been cleaned using an ammonium hydroxide/ hydrogen peroxide/water mixture $1: 1: 1$ at $68^{\circ} \mathrm{C}$ for $1 \mathrm{~h}$, then they were rinsed with millipore water and left overnight in water to stabilize the $\mathrm{SiO}_{2}$ top layer. The day after, they were dried and coated with the polymer film.

VIS interferometry: The film thicknesses of the as-prepared and post-treatment films were determined using a white-light interferometer (NanoCalc 2000, Ocean Optics).

Grazing-incidence small-angle X-ray scattering. GISAXS experiments were performed at HASYLAB beamline BW4 equipped with a MarCCD camera as an area detector [21]. The wavelength was $1.388 \AA$ and the sample-todetector distance $2.23 \mathrm{~m}$. The beam was focused by Beryllium compound refractive lenses to a size of $\sim 40 \mu \mathrm{m} \times 20 \mu \mathrm{m}$ (horizontal $\times$ vertical) at the sample position. In the experiment, the incident angle, $\alpha_{i}$, was chosen at $0.20^{\circ}-0.25^{\circ}$, thus at values slightly above the critical angle of total external reflection of $\mathrm{SiO}_{x}$ which is $\alpha_{c S}=0.19^{\circ}$ at the wavelength used. In addition to a point-like beamstop shielding the detector from the specularly reflected beam, a rod-like beamstop from tantalum was used to block the intense diffuse scattering in the scattering plane. The pixel size of the detector was $79.1 \mu \mathrm{m} \times 79.1 \mu \mathrm{m}$. $q_{\|}=\sqrt{q_{x}^{2}+q_{y}^{2}}$ and $q_{z}$ are the in-plane and the normal components of the scattering vector, respectively. For small incident angles and scattering angles, the coordinates of the 2D detector correspond approximately to $q_{y}$ and to $q_{z}$. In all images, the specularly reflected beam as well as the Yoneda peaks are expected. The latter are intensity maxima at exit angles $\alpha_{f}=\alpha_{C S}$ or $\alpha_{f}=\alpha_{C P}, \alpha_{C P}$ being the critical angle of total external reflection of the polymer film.

For randomly oriented lamellae, rings of high intensity around the direct beam (diffuse Debye-Scherrer rings, DDSRs) and around the specularly reflected beam are expected as well (Scheme 1) [17]. In contrast to transmission scattering, the intensity along the DDSR is not homogeneously distributed, but is maximum near the Yoneda peaks appearing at the $q_{z}$ values corresponding to the critical angles of the polymer film and the substrate (in the socalled Yoneda band) and decays towards high $q_{z}$ [22]. The lamellar thickness, $D_{l}$, is calculated using Bragg's law:

$D_{l}=\frac{2 \pi}{q_{y, D D S R}}$

where $q_{y, D D S R}$ is the radius of the DDSR centered at the specularly reflected beam. In the present case, the DDSRs are very weak and are only discernible from the background in the Yoneda band, thus their positions and radii cannot be read off at the $q_{z}$ value of the specularly reflected beam. Instead, the intensity profiles along $q_{y}$ were

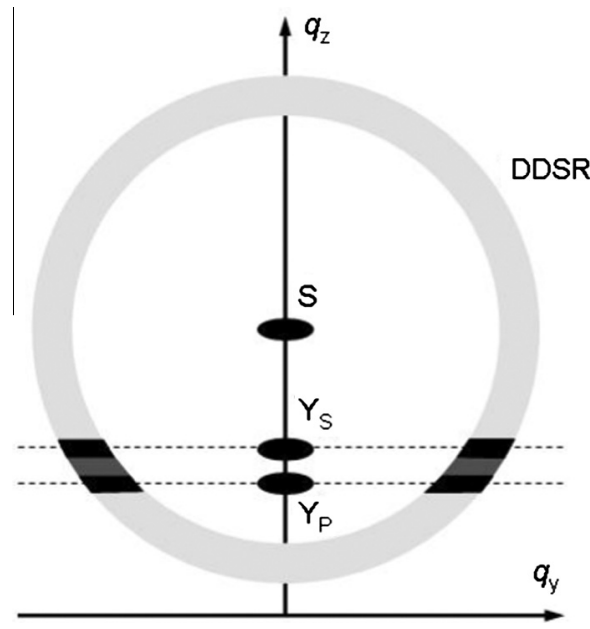

Scheme 1. Schematic representation of the scattering from a randomly oriented lamellar thin film. $S, Y_{S}$ and $Y_{P}$ denote the specularly reflected beam and the Yoneda peaks of the substrate and the polymer film, respectively. DDSR denotes the first-order diffuse Debye-Scherrer ring. The darker the ring, the higher its intensity. The dashed lines mark the $q_{z}$ positions of $Y_{S}$ and $Y_{P}$. Not shown is the strong intensity decay of the ring below $Y_{P}$. 
integrated over a stripe of a width along $q_{z}$ of $0.003 \AA^{-1}$ centered at the $q_{z}$ position of the Yoneda peak of the polymer, where the scattering is most intense (Scheme 1 ), namely at $q_{z}=0.028,0.027$ and $0.031 \AA^{-1}$ for the thicker, the intermediate and the thin film, which were measured at $\alpha_{i}=0.22^{\circ}, 0.20^{\circ}$, and $0.25^{\circ}$, respectively. Their $q_{y}$-range covers $-0.16 \AA^{-1}$ to $0.16 \AA^{-1}$. The peak positions were determined by fitting Lorentz functions to the peaks on both sides of the scattering plane, including scattering peaks due to the Kapton windows used in our chamber:

$$
\begin{aligned}
I\left(q_{y}\right)= & \frac{I_{1}}{1+4\left(\left(q_{y}-q_{y 1}\right) / w_{1}\right)^{2}}+\frac{I_{2}}{1+4\left(\left(q_{y}-q_{y 2}\right) / w_{2}\right)^{2}} \\
& +\frac{I_{D D S R}}{1+4\left(\left(q_{y}-q_{y, D D S R}\right) / w_{y, D D S R}\right)^{2}},
\end{aligned}
$$

where the first two terms describe the scattering of the Kapton exit windows of the flight tube, whereas the latter describes the first-order DDSR of the sample. $q_{y i}$ and $q_{y, D D S R}$ are the peak positions $(i=1,2), w_{i}$ and $w_{y, D D S R}$ the full widths at half maximum (fwhm) and $I_{i}$ and $I_{D D S R}$ the peak heights. For the precise determination of the $q_{y, D D S R}$ values, the fitting results from negative and positive $q_{y}$ values were averaged. The systematic overestimation in the determination of the repeat distance, $D_{l}$, by reading it off at the Yoneda position of $\mathrm{SiO}_{x}$ (Scheme 1 ) is estimated below $1 \AA$ at $\alpha_{i}=0.20^{\circ}$ (used for the intermediate film thickness), $3 \AA$ at $0.22^{\circ}$ (thick film) and $15 \AA$ at $0.25^{\circ}$ (thin film). This error only affects the absolute $D_{l}$ values; within each data set, the results are consistent. Also, the width of the ring is slightly overestimated.

The number of correlated repeat units in the film plane, $N_{S}$, was estimated from the expression for lamellae as follows [23]:

$N_{s} \cong \frac{2.75}{\pi} \times \frac{q_{y, D D S R}}{w_{y, D D S R}}$.

The lateral correlation length $\xi$ amounts to $N_{S} D_{l}$.

The conversion of the 2D images from pixels to $q$ values as well as the construction of 1D profiles and the peak fitting were carried out using the GISAXS Analysis Package, written by us within the Igor Pro development environment.

After alignment of the sample in the X-ray beam and GISAXS measurements of the as-prepared film, $2 \mathrm{ml}$ of $\mathrm{HX}$ were injected into a reservoir in the sample cell through a Teflon capillary, and GISAXS images were taken subsequently with an exposure time of $60 \mathrm{~s}$, while the film was immersed in saturated vapor. A custom-made vapor cell based on an aluminum cylinder equipped with a solvent reservoir was used to swell the film with $\mathrm{HX}$ vapor $[15,16]$. All measurements were carried out at room temperature (RT). The sample was kept in saturated vapor for $\sim 45 \mathrm{~min}$. Drying of the sample was carried out by flushing the sample cell with $\mathrm{N}_{2}$ gas. Again, GISAXS images were taken in real time with an exposure time of $60 \mathrm{~s}$. For the second vapor/drying cycle, the procedure was repeated. To avoid beam damage during the time-resolved GISAXS measurements, the sample was moved sideward after each measurement. After each vapor treatment and drying, the sample was realigned.
Atomic force microscopy (AFM): Tapping mode AFM experiments were carried out using a Jeol JSPM 5200 instrument with point-probe silicon SPM sensors, type CSC 12/AIBS/50 from $\mu$ Mash, having a typical resonance frequency of $150 \mathrm{kHz}$. A set-point ratio of $1.2-1.5$ was chosen. Due to their different viscoelastic properties, there is a strong phase contrast between POS and PBMA domains. A routine written by us within the IgorPro environment was used for Fourier transformation of the images. The 1D Fourier transforms are given in dependence on $k$, the wave number, which is an inverse length scale.

Samples for AFM images were prepared in the same way as the ones used for the GISAXS measurements and one vapor/drying cycle was carried out in the same way. AFM measurements were carried out after preparation and after one vapor/drying cycle.

\section{Results}

In a previous study [14] it was found using AFM that the surface of a fresh dip-cast film having a thickness of $500 \AA$ was featureless. When the thin film was swollen by HX and subsequently dried, perpendicular (standing) lamellae were observed at the surface. To elucidate the mechanisms of structure formation, we have carried out time-resolved GISAXS measurements on three $\mathrm{P}(\mathrm{OS}-b$-BMA) films having similar or lower film thicknesses. This way, we get an insight into the role of the film interfaces. First, we confirm that the as-prepared samples do neither reveal any surface structure nor any inner structure on the length scales investigated. Afterwards, we present the GISAXS results from the three films during repeated vapor/drying cycles as well as the surface structures measured after one vapor/drying cycle. Finally, we discuss the role of the film thickness on the structure formation process.

\section{Structures of the as-prepared samples}

The film thicknesses of the as-prepared samples as determined using VIS interferometry are $D_{f}=520 \pm 50 \AA$, $430 \pm 50 \AA$ and $350 \pm 50 \AA$ for films spin-coated at $1000 \mathrm{rpm}, 2000 \mathrm{rpm}$ and $3000 \mathrm{rpm}$, respectively; they amount to 2.0, 1.6 and $1.3 \times D_{l}^{\text {bulk }}$, respectively.

The AFM images after spin-coating and drying at room temperature do not show any evidence of surface order for the thick and the intermediate films (Fig. 1a and b). Only in the thin film, small and weakly ordered lamellar domains are present at the surface (Fig. 1c), thus, after spin-coating, lateral structures are already present at the film surface. (The phase images are featureless.) For none of the three films, the 2D Fourier transforms (insets in Fig. 1a-c) display distinctive features or do the corresponding 1D Fourier transforms show any peaks which, for lateral lamellar order, would be expected at $k=1$ / $260 \AA=0.0038 \AA^{-1}$; thus no long-range order is achieved after spin-coating [20]. The RMS surface roughnesses are $30 \AA, 20 \AA$ and $10 \AA$, for the thick, the intermediate and the thin film, respectively.

The GISAXS maps of the three as-prepared films (Fig. 1d-f) do not show any features apart from the specu- 
(a)

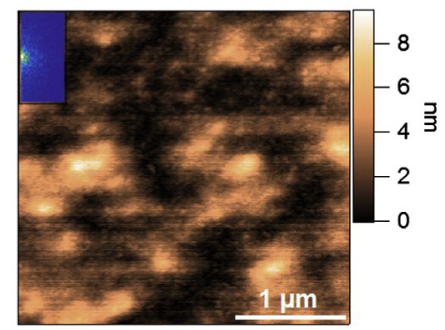

(d)

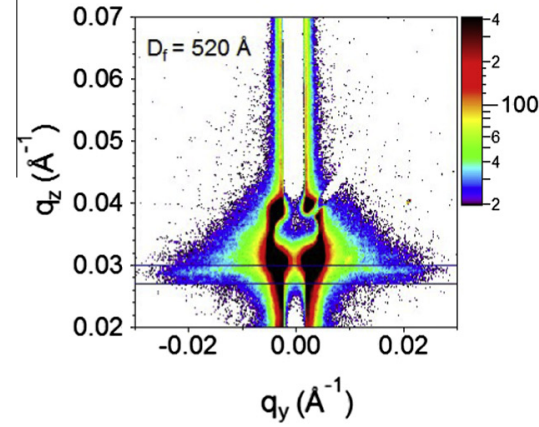

(g)

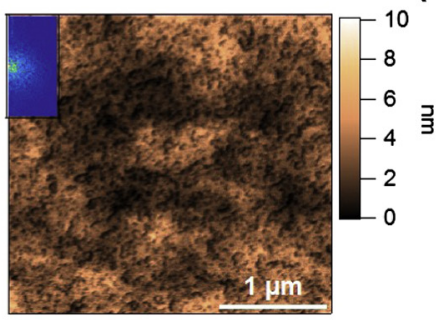

(b)

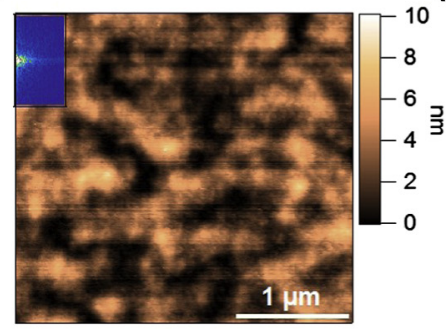

(c)

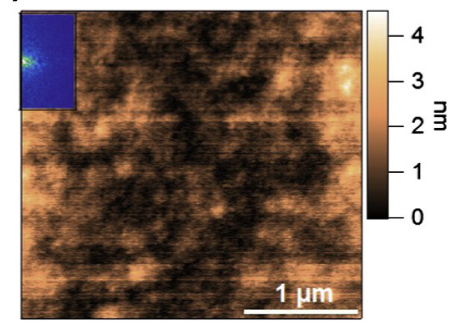

(e)

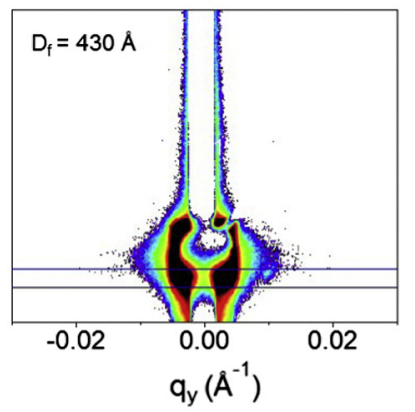

(h)

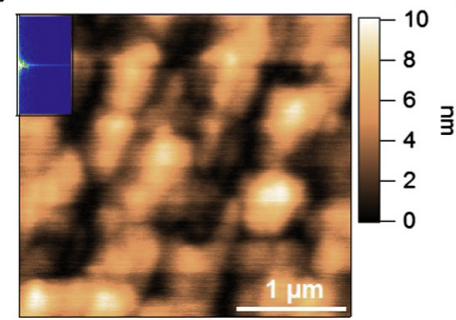

(f)

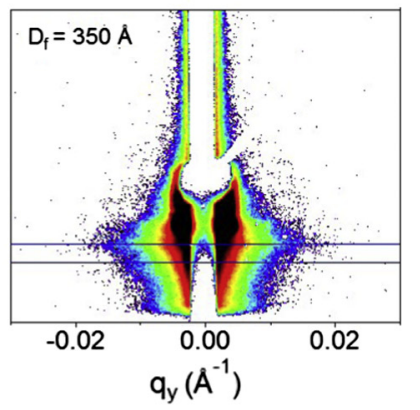

(i)

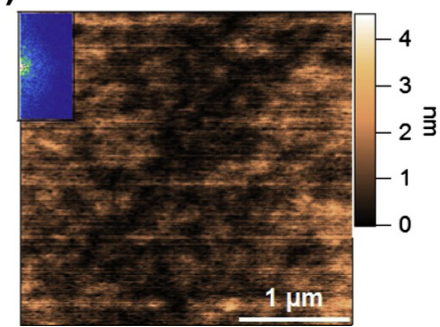

Fig. 1. Ex situ investigations. For each film thickness (given in the figure), three images are shown: AFM image of the as-prepared film (a-c), 2D GISAXS images of the as-prepared film (d-f), and AFM images after one vapor/drying cycle $(\mathrm{g}-\mathrm{i})$. The AFM height images have a size of $3 \mu \mathrm{m} \times 3 \mu \mathrm{m}$. The insets show the corresponding 2D Fourier transforms, ranging from -0.005 to $0.005 \AA^{-1}$. The color scale for all GISAXS pictures is the same as in Fig. $1 \mathrm{~d}$. The white stripes in the center are due to the beamstops. The blue horizontal lines in the GISAXS images show the regions used to construct the intensity profiles. (For interpretation of the references to colour in this figure legend, the reader is referred to the web version of this article.)

larly reflected beam and diffuse scattering, which is presumably due to surface roughness and to weak internal structuring. The first-order Bragg peak from a lamellar structure would be expected at $q_{y}=2 \pi / 260 \AA=0.024 \AA^{-1}$. The $1 \mathrm{D}$ intensity profiles (Fig. 2), obtained by integrating over a stripe having a $q_{z}$ width of $0.003 \AA^{-1}$ centered at the $q_{z}$-positions of the Yoneda peaks of the polymer films, however, do not show any peaks.

\section{Surface textures after vapor treatment with $H X$ and after drying}

To elucidate the formation of lateral structures by treatment with saturated HX vapor, we investigated the surface structures ex situ AFM after one vapor/drying cycle (Fig. 1g-i). The surface texture of the thick film after the first vapor/drying cycle shows a weak, small-scale structure (Fig. 1g). It is not regular enough to result in a peak in the 1D Fourier transform, though. The RMS surface

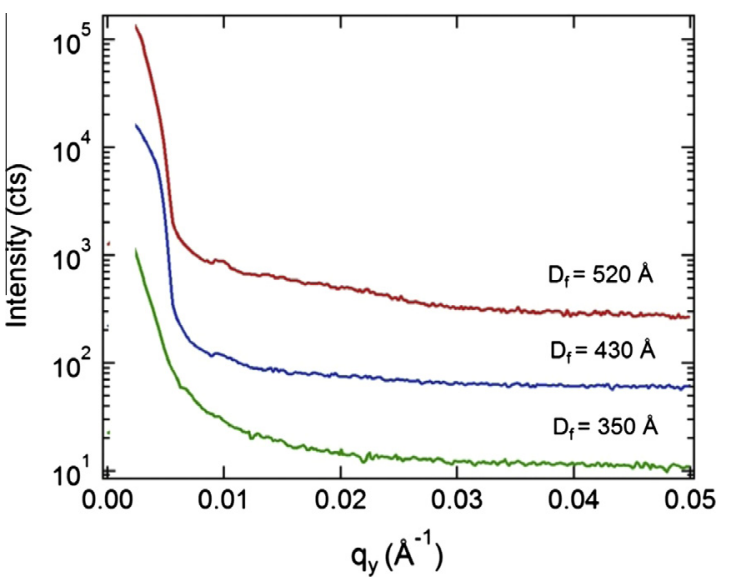

Fig. 2. As-prepared films. 1D GISAXS intensity profiles along $q_{y}$ for the three different thicknesses. The film thicknesses are given for each profile. The curves are shifted vertically. 


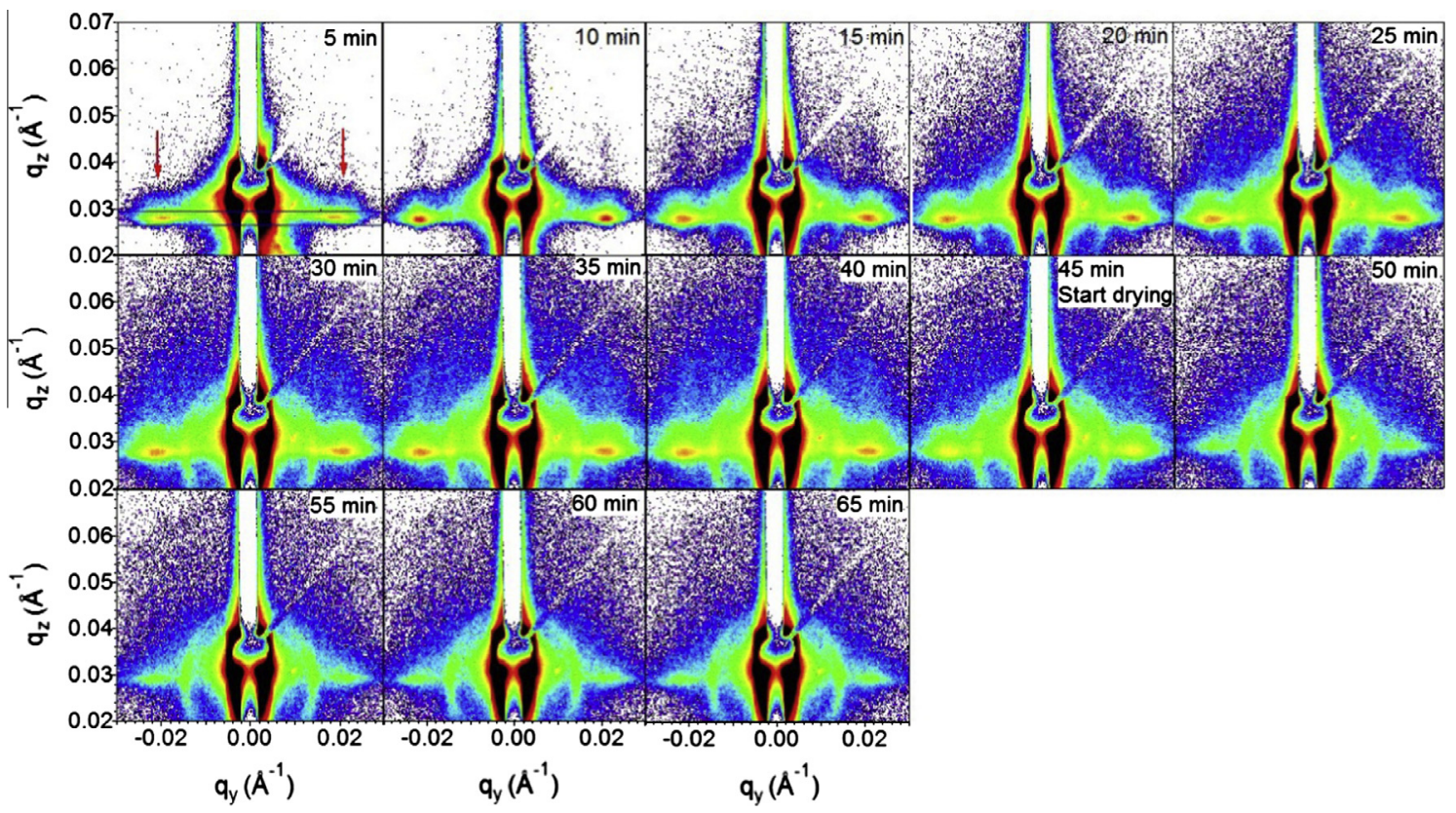

Fig. 3. Representative GISAXS images of the thick film during treatment with HX vapor and subsequent drying, taken at $\alpha_{i}=0.22^{\circ}$. The times after injection of HX into the cell are given in each image. Drying starts after 45 min by starting a flow of $\mathrm{N}_{2}$ gas. The arrows mark the DDSRs. The ring-like features are due to scattering from Kapton. For all images, the color scale and the regions used to construct the intensity profiles are the same as in Fig. $1 \mathrm{~d}$.

roughness is $15 \AA$, the surface is thus smoother than in the as-prepared film. In contrast to the thick film, large surface structures appear in the film with intermediate thickness after vapor treatment and drying (Fig. 1h). The Fourier transform does not show any peak, though. The absence of a structural peak indicates that the large structures appearing at the top of the film surface are due to irregular inhomogeneities of the film surface, induced by the swelling/drying cycle. The RMS surface roughness is now $25 \AA$, thus higher than in the as-prepared film. At the surface of the thin film, small structures appear after one vapor/ drying cycle (Fig. 1i). These surface features may be due to slightly better defined lateral structure, as they are already present in the as-prepared film. The $2 \mathrm{D}$ Fourier transform shows, however, no feature. Therefore, longrange order of the lateral structures present at the surface is not achieved after vapor treatment. At the same time, the surface orientation of the lateral structure, as it is already present after spin-coating, is not drastically affected by the vapor treatment. The RMS surface roughness is $10 \AA$, thus unchanged from the as-prepared film. The AFM images of the three films are thus not conclusive of the possible internal structural changes during the vapor treatment and drying since only weak and irregular structures are present. Therefore, GISAXS is used in order to probe the internal structure of the film.

\section{Morphologic changes during vapor treatment with $\mathrm{HX}$ and after drying}

The processes going on during one or two vapor/drying cycles were monitored using time-resolved GISAXS. In these experiments, vapor treatment was started by injecting 1-2 $\mathrm{ml}$ of $\mathrm{HX}$ into the reservoir at the bottom of the sample cell $(\sim 1-2 \mathrm{~cm}$ below the sample). After $\sim 45 \mathrm{~min}$, drying was started by flushing the cell with dry $\mathrm{N}_{2}$ gas.

Thick film: The changes in the thick film during exposure to saturated HX vapor are evident from the time series of GISAXS images shown in Fig. 3. After injection of liquid HX into the cell, out-of-plane peaks appear at $q_{y}=0.02 \AA^{-1}$ and become more intense with time, i.e. an increasing fraction of the film contributes to the scattering from a lateral structure. The peak intensity keeps growing until the end of the vapor treatment. We attribute these peaks to the enhanced part (Yoneda band) of the first-order DDSR due to a randomly oriented lamellar structure (Scheme 1 ). The repeat distance of the lateral structure, $D_{l}$, is determined by fitting Eq. (2) to the 1D profiles constructed as described in the experimental section (Fig. 4). These profiles show an intensity maximum due to the DDSR, which becomes significantly more intense after $\sim 8$ min and which changes position in a complex way with time. During drying, the peak weakens immediately (Figs. 3 and 4).

From the $q_{y}$ position of the peak, we calculate the repeat distance, $D_{l}$, as a function of time (Fig. 5a). It becomes possible to read off a peak position after $1 \mathrm{~min}$ of vapor treatment. At that time, $D_{l}$ amounts to $314 \AA$ (Eq. (1)); this value is already significantly larger than $D_{l}^{\text {bulk }}(260 \AA)$ [14]. During the first $10 \mathrm{~min}, D_{l}$ decreases until it reaches $293 \pm 2 \AA$, then stabilizes after a small overshoot at $297 \pm 2 \AA$ after $21 \mathrm{~min}$. It remains nearly unaffected by the drying process with the final value being $296 \pm 2 \AA$, which is still higher than $D_{l}^{\text {bulk}}$; indicating remaining solvent in the film. The number of repeat units, $N_{S}$, in the film plane (Eq. (3)), increases from $\sim 1.3$ and reaches a maxi- 


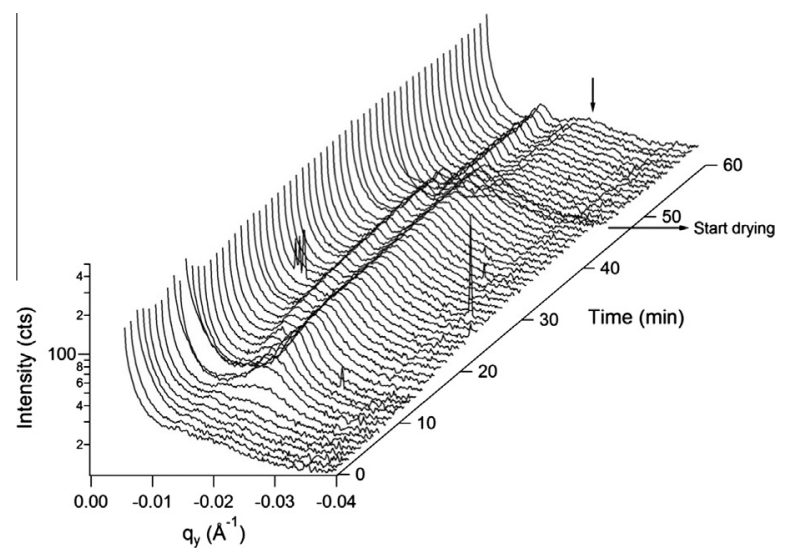

Fig. 4. Thick film during the first cycle of vapor treatment and subsequent drying. Intensity profiles along $q_{y}$ as a function of time, centered at $q_{z}=0.028 \AA^{-1}$. The vertical arrow indicates the position of the first-order DDSR. An additional peak at a lower $q_{y}$ value of $0.014 \AA^{-1}$ is due to scattering from the Kapton windows. The drying starts at the marked position.

mum of $N_{S}=4$ after $5 \mathrm{~min}$, then slowly decreases and stabilizes at a value of $\sim 2$ after $21 \mathrm{~min}$ (Fig. 5b). The value of $N_{S}$ decreases abruptly to a value of 1.4 upon drying. Even though the DDSRs are weak during drying, they are still present, i.e. the lateral orientation is partially maintained within the film. The second vapor/drying cycle shows that the behavior of both $D_{l}$ and $N_{S}$ during the first cycle is qualitatively reproducible.

Thus, probing the internal structure of the thick film, we observe a tendency to a formation of a lateral structure, possibly the lamellar morphology. This structure is most ordered after 5 min when the number of correlated repeat units is maximum. Further vapor treatment results in a partial deterioration of the structure. Moreover, upon drying, the structure is only partially stable which explains why the resulting surface texture is only weak (Fig. 1g). The solvent evaporation process during drying is presumably faster near the film surface than inside the films, thus the lamellar structure near the film surface has more defects than inside the film [20]. The lateral repeat distance shows relatively fast kinetics with the changes being finished within $\sim 20$ min upon vapor treatment and $\sim 5 \mathrm{~min}$ upon drying.

Intermediate film: During exposure to saturated HX vapor and drying, the GISAXS maps of the $\mathrm{P}(\mathrm{OS}-b$-BMA) film with $D_{f}=430 \AA$ (Fig. 6) shows qualitatively the same behavior as the thick film. However, the changes are slightly faster than in the thick film. The DDSR becomes more intense during the vapor treatment, i.e. the fraction of the film displaying a lateral structure increases with time. In contrast to the thick film, the growth of the peak intensity finishes already after $\sim 20 \mathrm{~min}$. When drying is started, the DDSR weakens immediately. A second cycle of vapor treatment and subsequent drying was not performed due to beam time restrictions.

The $1 \mathrm{D}$ intensity profiles as well as the resulting fitting parameters (Figs. 7 and 8) show qualitatively very similar behavior to the one of the thick film. However, the time and length scales are different - the minimum of $D_{l}$ is reached already after $8 \mathrm{~min}$, thus earlier than in the thick film, and the final value of $D_{l}$ in the vapor is with $289 \pm 2 \AA$ slightly lower than in the thick film but still higher than in the bulk. Also the overshoot of $N_{S}$ to a value $N_{S}=3$ takes place earlier (after $5 \mathrm{~min}$ ) than in the thick film. $N_{S}$ reaches a final value of $\sim 2$, which is similar to the value in the thick film. The height of the peak settles after $\sim 20 \mathrm{~min}$ and does not continue to grow as in the thick film.

Upon drying, the peak nearly vanishes, therefore no further fitting is possible.

Thin film: During exposure to saturated HX vapor, the thin film shows a behavior completely different from the previous ones. The GISAXS maps of the P(OS-b-BMA) film with $D_{f}=350 \AA$ show a very weak DDSR, much weaker than the ones observed in the intermediate and the thick films, because the lower film thickness gives rise to less scattering (Fig. 9). The DDSR keeps becoming more intense during the first $\sim 30 \mathrm{~min}$ and then stops growing. Upon drying, the DDSR weakens immediately, as in the two thicker films.

The 1D intensity profiles show that the intensity of the peaks due to the DDSR starts to increase only after $\sim 10 \mathrm{~min}$ (Fig. 10). The behavior of $D_{l}$ is very different from the one in the two thicker films: During the first $30 \mathrm{~min}, D_{l}$

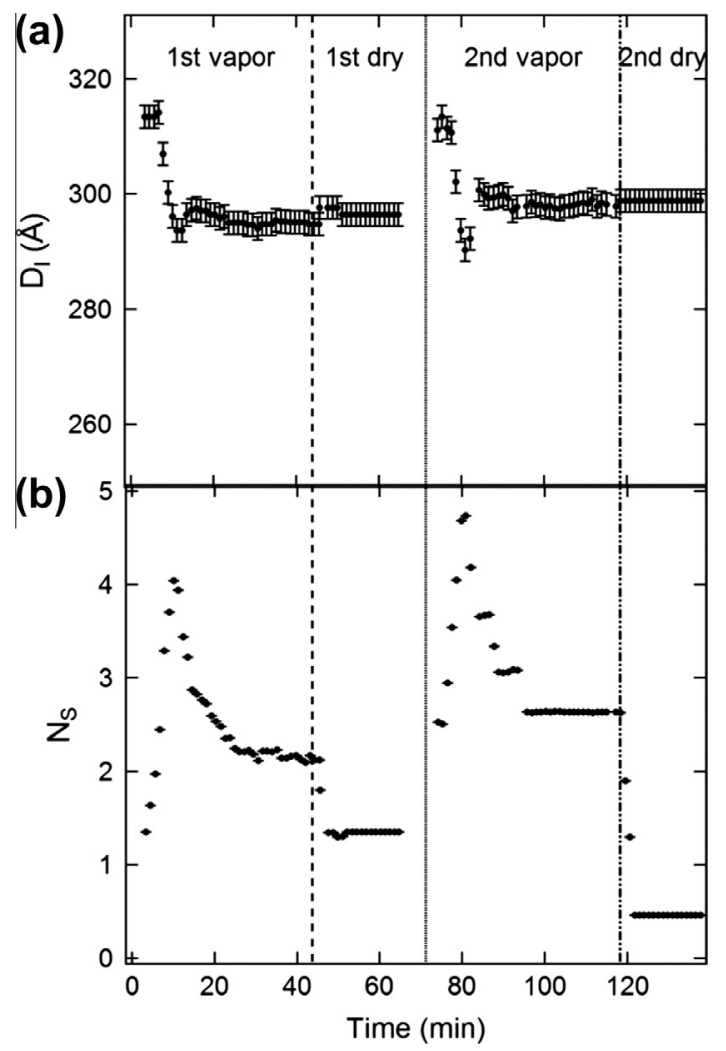

Fig. 5. GISAXS results of the thick film. (a) Lateral repeat distance, $D_{l}$, and (b) number of correlated repeat units, $N_{S}$, as a function of treatment time. The dashed, dotted and dash-dotted lines indicate the start of the first drying, the second vapor treatment and the second drying. 


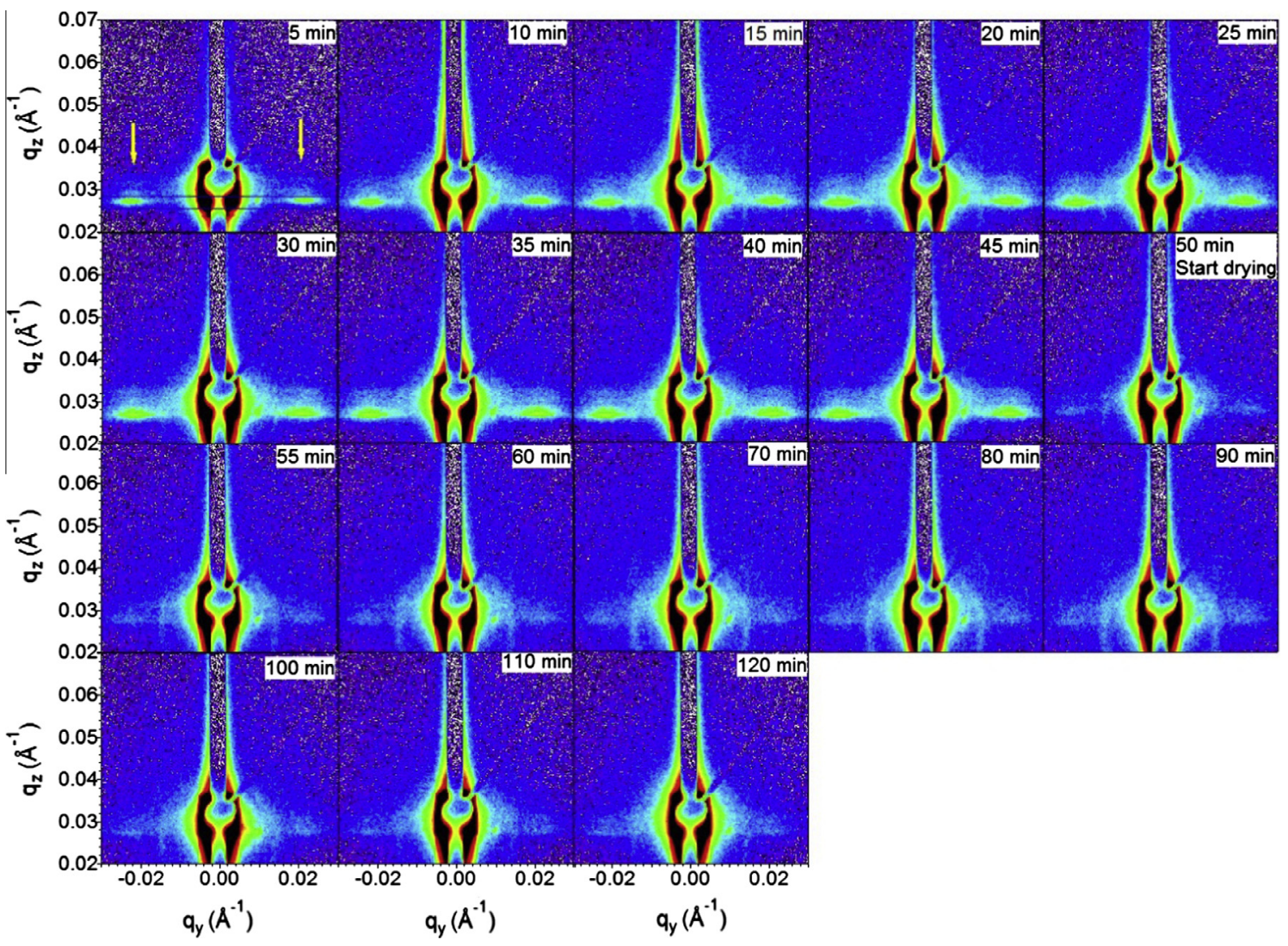

Fig. 6. Representative GISAXS images of the film with intermediate thickness during treatment with HX vapor and subsequent drying, taken at $\alpha_{i}=0.20^{\circ}$. Same scale and notation as in Fig. 3.

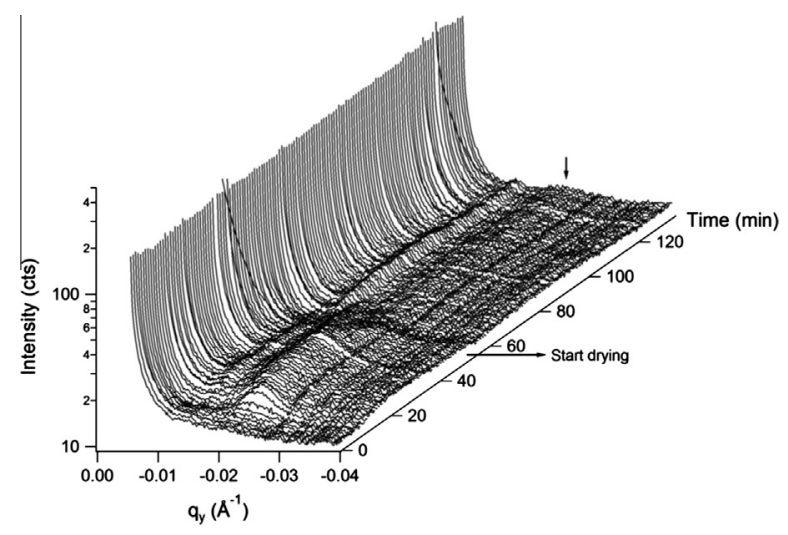

Fig. 7. Intermediate film during the first cycle of vapor treatment and subsequent drying. Intensity profiles along $q_{y}$ as a function of HX vapor treatment time and subsequent drying, centered at $q_{z}=0.027 \AA^{-1}$. Same notation as in Fig. 4.

increases slowly until it reaches a broad maximum at $296 \pm 2 \AA$ after $40 \mathrm{~min}$ and decreases to a value of $289 \pm 2 \AA$ at the end of the vapor treatment (Fig. 11a). These changes are much slower than in the intermediate and the thick film. During the first 9 min of vapor treatment, $N_{S}$ increases to $\sim 1.5$ during the first $\sim 10 \mathrm{~min}$, then decreases to very low values (Fig. 11b).
Upon drying, $D_{1}$ keeps decreasing smoothly to $272 \pm 2 \AA$. Taking into account that, for this film, $D_{1}$ is overestimated by $\sim 15 \AA$ (Scheme 1 ) the final value is similar to $D_{l}^{\text {bulk }}$. After $\sim 10$ min of vapor treatment, the peak becomes very weak but is still present (Fig. 10, inset). Thus, the inner structure of the thin film rearranges and the bulk lamellar thickness is reached. $N_{S}$ decreases abruptly upon drying to a value of $\sim 0.8$. The second vapor/drying cycle is completely different from the first one, possibly because, due to the thinness of the film, the film surface and the film/substrate interface have a kind of pinning effect, which is stronger than in the intermediate and the thick film. Moreover, the thin film is more prone to dewetting. This may also explain why the bulk lamellar thickness is assumed.

In the case of the thin film, the AFM image features lateral structure at the surface, both before and after treatment, whereas GISAXS does not provide any evidence of lateral structure within the film after spin-coating. During solvent vapor annealing, GISAXS provides in situ evidence of a lateral structure appearing also within the film. Thus, the ordering starts from the surface, slowly propagating within the film.

\section{Conclusions}

Thin films from lamellar $\mathrm{P}(\mathrm{OS}-b$-BMA) diblock copolymers are very susceptible to the quality and selectivity of 


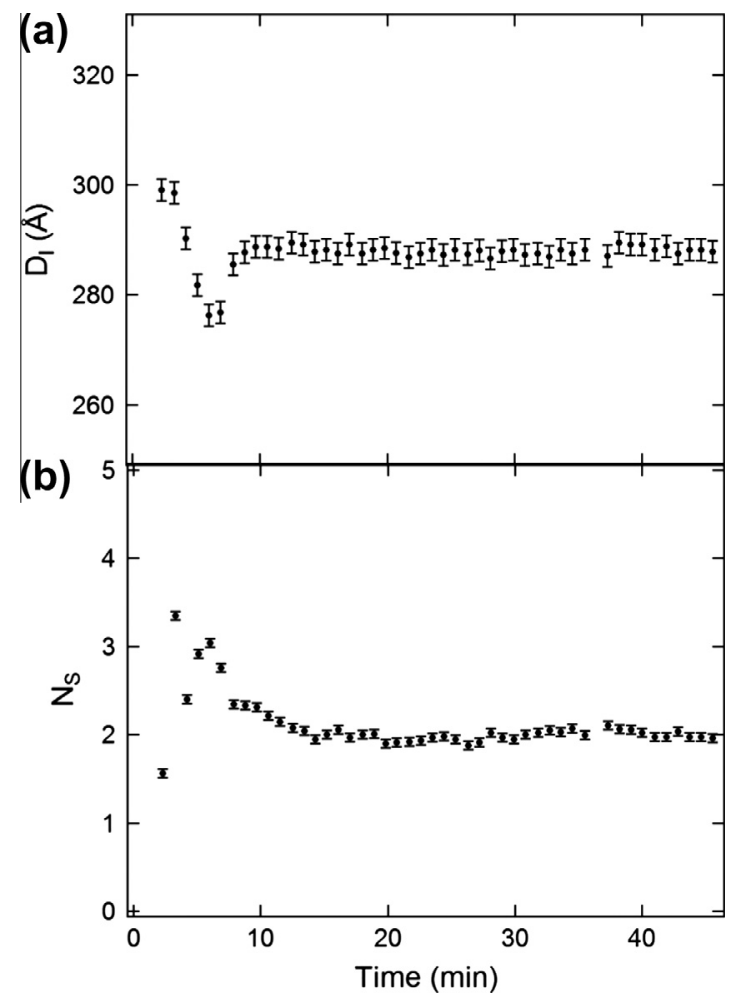

Fig. 8. GISAXS results of the film with intermediate thickness. (a) Lateral repeat distance, $D_{l}$, and (b) number of correlated repeat units, $N_{S}$, as a function of treatment time (only vapor treatment). the solvent. For instance, $\mathrm{HX}$ is a solvent which is close to non-selective and rather poor for both blocks. At room temperature, the POS block is above and the PBMA block is only slightly below its glass transition temperature, thus vapor treatment of the $\mathrm{P}$ (OS- $b$-BMA) system is expected to have a relatively fast effect on the structure, as previously observed [14]. Indeed, we could confirm that, on time scales similar to the ones used in the present study, thin films from poly(styrene- $b$-(butyl methacrylate)) do not show any changes in scattering during vapor treatment with HX. Thus, exchanging poly(4-octylstyrene) by (glassy) polystyrene hampers the structural reorganizations, most probably because of the high glass transition temperature of polystyrene $\left(\sim 100^{\circ} \mathrm{C}\right)$ as well as its insolubility in HX. We conclude that, to achieve lateral structures, both blocks need to have a high mobility.

Our study shows that lateral structures can be obtained most rapidly for the intermediate film thickness $(430 \AA$, $\left.D_{f}=1.6 \times D_{l}^{\text {bulk }}\right)$. In the thick film $\left(D_{f}=2.0 \times D_{l}^{\text {bulk }}\right)$, the same behavior with an undershoot of $D_{l}$ is observed, however, on a slightly longer time scale. In these two films, the lateral structure is partially preserved during drying. On the contrary, the thin film $\left(D_{f}=1.3 \times D_{l}^{\text {bulk }}\right)$ shows very different and very slow kinetics in HX vapor, and eventually, the bulk lamellar thickness is approximately reached. The restructuring of this film may be slowed down by the more pronounced effect of the film surface and the substrate surface compared to the intermediate and the thick film, and it may be accompanied by an incipient dewetting from the substrate. A long time is thus needed in the thin film to rearrange the originally disordered polymer material, within the inner part of the film, into an ordered structure.

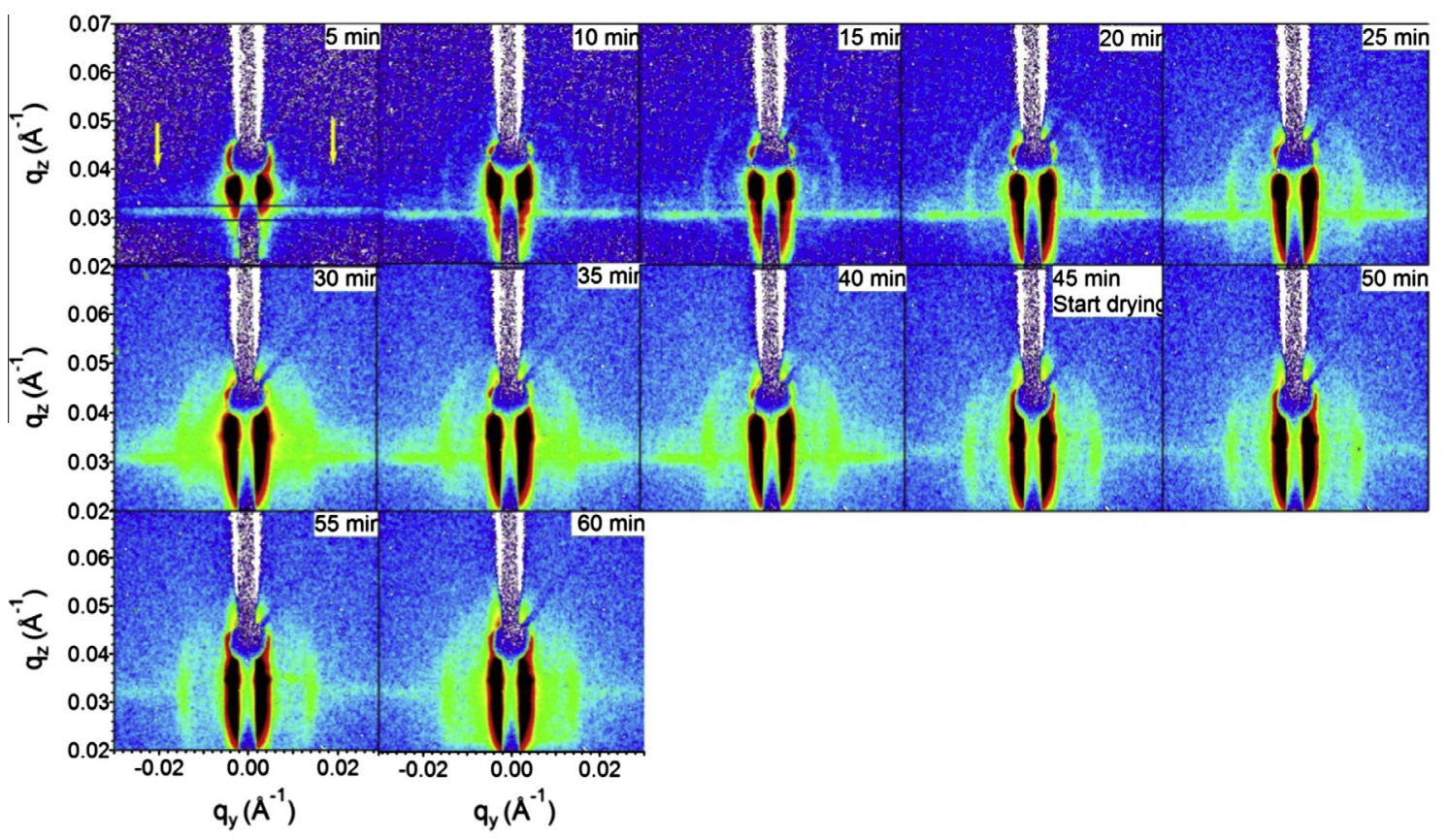

Fig. 9. Representative GISAXS images of the thin film during treatment with HX vapor and subsequent drying, taken at $\alpha_{i}=0.25^{\circ}$. Same scale and notation as in Fig. 3. 


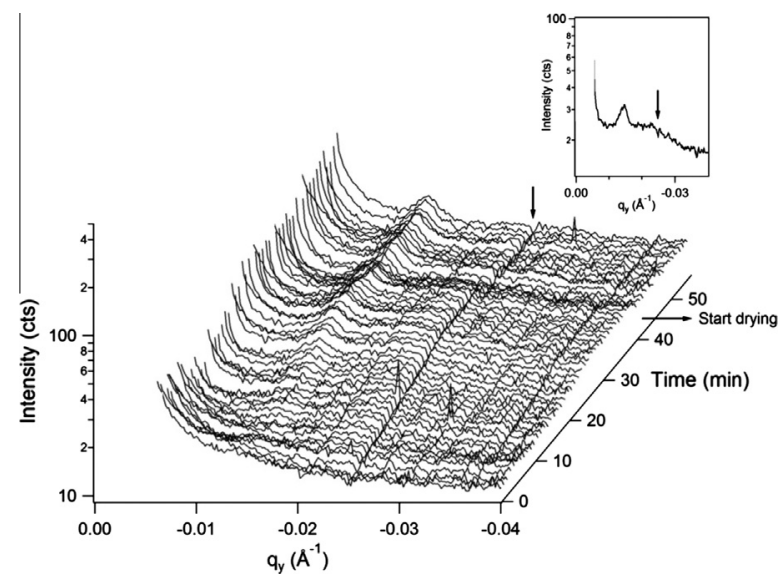

Fig. 10. Thin film during the first cycle of vapor treatment and subsequent drying. Intensity profiles along $q_{y}$ as a function of HX vapor treatment time and subsequent drying, centered at $q_{z}=0.031 \AA^{-1}$. The inset shows the intensity profile after $20 \mathrm{~min}$ of drying, the arrow indicates the position of the first-order DDSR. Same notation as in Fig. 4.

Moreover, the decrease of the value of the final $D_{l}$ value after drying with decreasing film thickness points to the fact that the amount of remaining solvent in the film decreases with decreasing film thickness and that the system approaches a configuration with a lateral repeat distance close to the bulk lamellar thickness. We conclude that, in thin films, controlling the flow rate of the solvent vapor

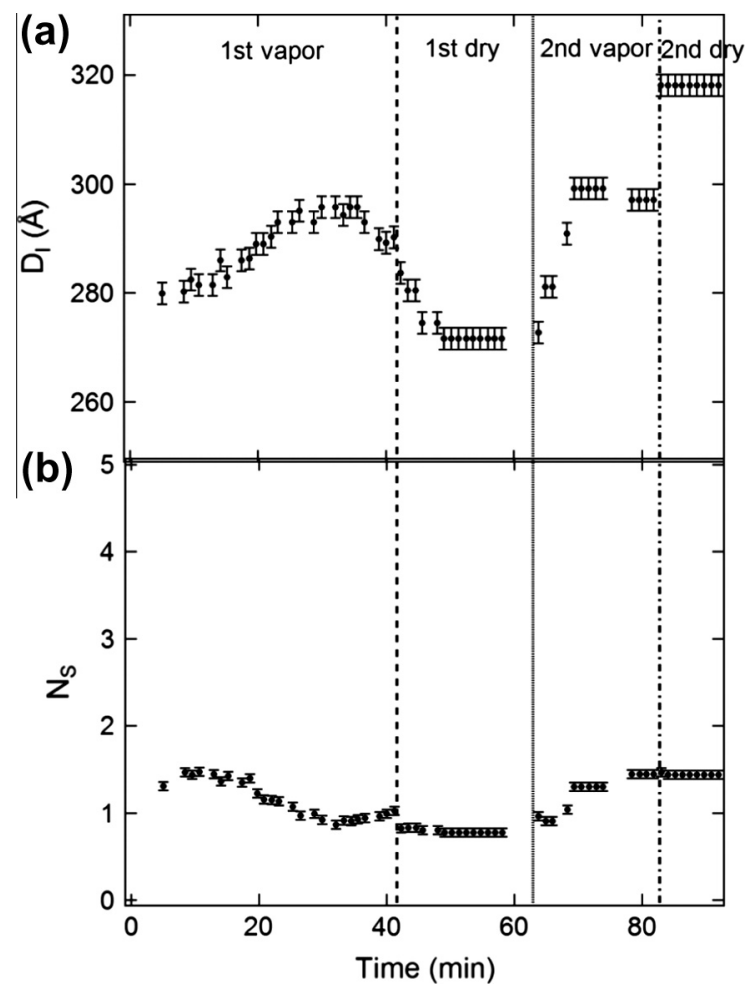

Fig. 11. GISAXS results of the thin film. (a) Lateral repeat distance, $D_{l}$, and (b) number of correlated repeat units, $N_{S}$, as a function of treatment time. as well as a slower drying, may improve the formation of the lateral structure throughout its thickness, thus propagating the self-assembly process within the whole film thickness, as it commences at the surface after spincoating.

The changes of the orientation of the morphology observed upon vapor treatment and drying are consistent with the lateral structures observed previously after HX vapor treatment and drying using AFM [14]. Thus, for diblock copolymers with both blocks being above or close to the glass transition temperature, it is sufficient that the solvent used for treatment is a poor solvent (i.e. better than a non-solvent) in order to alter the lateral structure orientation, instead of e.g. thermo-annealing of thin layers to suitable temperatures as demonstrated in Ref. [24]. The present real-time, in situ GISAXS investigations elucidate the time scales of the inner structural changes within the entire film thickness, in contrast to methods like transmission electron microscopy (TEM), which are used to inspect the internal structure of polymer thin films, are limited to stable structures [25]. Neither is ex situ, post-treatment AFM not sufficient to reveal these processes since it only monitors the surface texture.

\section{Acknowledgments}

We thank A. Timmann, T. Hugel and B. Balzer for their help and for discussions. Financial support by the International Graduate School Materials Science of Complex Interfaces" (A.S.) is gratefully acknowledged. Portions of this research were carried out at the light source DORIS III at DESY. We thank DESY for providing beamtime and excellent equipment. DESY is a member of the Helmholtz Association (HGF). We thank GACR for financial support (P208/ 10/1600).

\section{References}

[1] Hamley IW. Nanotechnology 2003;14:R39.

[2] Segalman RA. Mater Sci Eng R 2005; 48: 191.

[3] Park M, Harrison C, Chaikin PM, Register RA, Adamson DH. Science $1997 ; 276: 1401$.

[4] Tokarev I, Krenek R, Burkov Y, Schmeisser D, Sidorenko A, Minko S, Stamm M. Macromolecules 2005;38:507.

[5] Sperschneider A, Schacher F, Gawenda M, Tsarkova L, Müller AHE, Ulbricht M, et al. Small 2007;3:1056.

[6] Yang SY, Park J, Yoon J, Ree M, Jang SK, Kim JK. Adv Funct Mater 2008;18:1371.

[7] Albalak RJ, Capel MS, Thomas EL. Polymer 1998;39:1647.

[8] Fukunaga K, Hashimoto T, Elbs H, Krausch G. Macromolecules $2002 ; 35: 4406$.

[9] Kim SH, Misner MJ, Xu T, Kimura M, Russell TP. Adv Mater $2004 ; 16: 226$

[10] Xuan Y, Peng J, Cui L, Wang H, Li B, Han Y. Macromolecules 2004;37:7301.

[11] Ludwigs S, Schmidt K, Krausch G. Macromolecules 2005;38:2376.

[12] Bang J, Kim SH, Drockenmuller E, Misner MJ, Russell TP, Hawker CJ. JACS 2006;128:7622.

[13] Peng J, Han Y, Knoll W, Kim DH. Macromol Rapid Commun 2007;28:1422.

[14] Černoch P, Štěpánek P, Pleštil J, Šlouf M, Sidorenko A, Stamm M. Eur Polym J 2007;43:1144.

[15] Papadakis CM, Di Z, Posselt D, Smilgies D-M. Langmuir 2008;24:13815.

[16] Di Z, Posselt D, Smilgies D-M, Papadakis CM. Macromolecules 2010;43:418.

[17] Busch P, Posselt D, Smilgies D-M, Rauscher M, Papadakis CM. Macromolecules 2007;40:630. 
[18] Smilgies D-M, Li R, Di Z, Darko C, Papadakis CM, Posselt D, Mater Res Soc Symp Proc 2009; 1147: 0001.

[19] Di Z, Posselt D, Smilgies D-M, Li R, Rauscher M, Potemkin II, et al. Macromolecules 2012;45:5185.

[20] Sepe A, Hoppe ET, Jaksch S, Magerl D, Zhong Q, Perlich J, et al. J Phys Condens Matter 2011;23:254213.

[21] Roth SV, Döhrmann R, Dommach M, Kuhlmann M, Kröger I, Gehrke R, et al. Rev Sci Instrum 2006;77:085106.
[22] Busch P, Rauscher M, Smilgies D-M, Posselt D, Papadakis CM. J Appl Cryst 2006:39:433

[23] Papadakis CM, Almdal K, Mortensen K, Posselt D. J Phys II France 1829;1997:7.

[24] Morkved TL, Jaeger HM. Euro Phys Lett 1997;40:643.

[25] Radzilowski LH, Carvalho BL, Thomas EL. J Polym Sci B Polym Phys 1996;34:3081. 